\title{
BMI, Blood Pressure, and Plasma Lipids among Centenarians and Their Offspring
}

\author{
Peng-Cheng Hai, ${ }^{1}$ Dong-Xiao Yao, ${ }^{2}$ Rui Zhao, ${ }^{3}$ Chen Dong, ${ }^{3}$ Sara Saymuah, ${ }^{4}$ \\ Yue-Song Pan, ${ }^{2}$ Zhi-Feng Gu, ${ }^{3}$ Yi-Long Wang, ${ }^{2}$ Chen Wang $\mathbb{D}^{1,5}$ and Jian-Lin Gao $\mathbb{D}^{3}$ \\ ${ }^{1}$ School of General Practice and Continuing Education, Capital Medical University, Fengtai, No. 10, \\ You An Men Wai Xi Tou Tiao, Beijing 100069, China \\ ${ }^{2}$ Department of Neurology, Beijing Tiantan Hospital, Capital Medical University, Beijing, China \\ ${ }^{3}$ Research Center of Gerontology and Longevity, Affiliated Hospital of Nantong University, Nantong, China \\ ${ }^{4}$ Department of Neurosurgery, Wayne State University School of Medicine, Detroit, USA \\ ${ }^{5}$ Department of General Practice, Beijing Tiantan Hospital, Capital Medical University, Beijing, China
}

Correspondence should be addressed to Chen Wang; wangchentr@gmail.com and Jian-Lin Gao; j14486@ntu.edu.cn

Received 7 October 2021; Accepted 30 December 2021; Published 20 January 2022

Academic Editor: Shuzhen Guo

Copyright (c) 2022 Peng-Cheng Hai et al. This is an open access article distributed under the Creative Commons Attribution License, which permits unrestricted use, distribution, and reproduction in any medium, provided the original work is properly cited.

\begin{abstract}
Background. The burden of cardiovascular diseases (CVDs) is increasing substantially due to population growth and aging. Determining effective prevention and understanding the underlying mechanisms remain desirable pursuits for increasing the quality of life. As centenarians and their offspring may have genetic advantages, they may present with healthier cardiovascularrelated profiles. Methods. We launched a cross-sectional household-based survey of centenarian families, including 253 centenarians, 217 centenarian offspring, and 116 offspring spouses without centenarian parents from county-level Chinese longevity city Rugao. Among offspring and offspring spouses were the following arrangements: 101 paired offspring and offspring spouses who lived together, 116 unpaired offspring, and 16 unpaired spouses. We investigated their cardiovascular-related health status including waist circumference, body mass index (BMI), blood pressure, and plasma lipids and compared results among centenarians, centenarian offspring, and offspring spouses. Results. Centenarians ranged from 99 to 109 years with a median age of 100 years. Centenarian offspring, with a median age of 70 years, and offspring spouses, with a median age of 69 years, shared similar age. Results of blood pressure, plasma lipid levels, and BMI displayed no significant difference between centenarian offspring and offspring spouses. However, centenarians appeared to have lower waist circumference, BMI, TC, LDL-C, TG, and diastolic blood pressure but higher levels of systolic blood pressure $(p<0.05)$. Multivariate analysis showed the prevalence of obesity, hypertension, and dyslipidemia was similar between centenarian offspring and offspring spouses, while centenarians appeared to have a lower prevalence of obesity and a higher prevalence of hypertension $(p<0.05)$. Conclusions. Centenarians and centenarian offspring did not present healthier BMI, blood pressure, or plasma lipids than offspring spouses. Further research on longevity and cardiovascular diseases are desirable.
\end{abstract}

\section{Introduction}

Cardiovascular diseases (CVDs) are the leading cause of disease burden and deaths in the world [1]. Due to population growth and aging, the burden of CVDs is increasing substantially in most low- and middle-income countries [1]. It is desirable to determine the underlying mechanisms for the development of feasible CVD prevention and to promote the ideal cardiovascular status and healthy aging [2].
Dyslipidemia, hypertension, diabetes, abdominal obesity, smoking, intake of fruits, vegetables, and alcohol, physical activity, and psychosocial factors account for over $90 \%$ of population attributable risks of myocardial infarction in the world [3]. The American Heart Association defined ideal health behaviors and factors including nonsmoking, adequate physical activity, healthy diet, normal body mass index (BMI), blood pressure, total cholesterol (TC), and fasting blood glucose [4]. Cardiovascular health-related 
factors, such as BMI, blood pressure, and lipids are associated with a variety of factors such as genes, age, diet, physical activity, and geographic environment [5-10]. Host genetics appear to influence personal responsiveness to dietary fats and cholesterol, and some risk alleles are found associated with hypertension, dyslipidemia, and obesity $[6,7,9,11]$. The prevalence of vascular diseases dramatically increases with age [12]. Total cholesterol and triglyceride (TG) concentrations increase between 20 and 80 years of age but decrease in centenarians [13]. On the other hand, the contribution by environmental factors are increasingly recognized $[1,14,15]$ : environmental exposures such as ambient air pollution, noise pollution, psychosocial, and economic stressors trigger risks for CVDs through various pathways [15]. Besides, familial environments affecting health-related behaviors play an important role. Hypertension, dyslipidemia, and obesity can be prevented by consuming a healthy diet including a limited intake of salt with adequate fruits and vegetables and engaging in healthy behaviors such as adequate physical activity and avoiding excessive alcohol intake [16-18].

Longevity and resistance to diseases such as CVDs may be regulated by shared underlying mechanisms [5]. There is genetic overlap between longevity and CVDs, where among 300 genes related to longevity, 87 are associated with cardiovascular-related traits including hypertension, lipid metabolism, energy metabolism, inflammation, and coagulation [5, 19-23]. Currently, there are many studies on centenarians and their offspring due to their perception as informative models for both longevity and CVDs. Studies revealed that centenarians and their offspring avoided or postponed the onset and progression of major CVDs [5, 24-26]. As for major cardiovascular-related factors such as BMI, blood pressure, and lipids, centenarians and their offspring seem to have genetic advantages with few risk variants and more protective variants $[19,21,23,27-29]$. However, from the perspective of clinical phenotype, studies presented inconsistent results. Centenarians may have a lower prevalence of hypertension, but lower blood pressures are associated with poor cognitive function [30]. Lipids of centenarians from diverse geographical regions have been investigated, and inconsistent results showed that plasma levels of total cholesterol, low-density lipoprotein cholesterol (LDL-C), high-density lipoprotein cholesterol (HDL-C), and TG were either normal, lower, or not different than elderly controls [31-33]. Lower BMI was observed among centenarians, suggested as one of the keys for longevity, and may be due to their eating habits including low consumption of red meat and cholesterol [34-36]. With respect to centenarian offspring, they presented a lower prevalence of hypertension, dyslipidemia, and obesity in some studies, while other studies found no significant difference [37-41]. Environmental factors affect gene expression and may contribute to the heterogeneity of cardiovascular-related profiles among centenarians and their offspring $[5,42]$. However, most studies were case-controls comprising of either centenarians and younger controls or centenarian offspring and age-matched controls; there are few studies investigating the health status of centenarian families including centenarians, centenarian offspring, and offspring spouses within the same environment.

As centenarians and their offspring may have genetic advantages, we hypothesized this population may present with healthier cardiovascular profiles. Therefore, we proposed to objectively determine cardiovascular health using cardiovascular-related health factors among recruited centenarians, their offspring, and offspring spouses without centenarian parents residing within the same environment to minimize the influence of environmental factors and better determine the extent of genetic components on CVD development and prevalence.

\section{Study Design and Participants}

The investigation was launched in Rugao, a county-level city in Jiangsu province in southern China. Rugao is renowned as a longevity city with the largest number of centenarians among county-level cities in China [43]. According to the data from the local Civil Affairs Bureau, Rugao had 394 centenarians in 2019.

Data were collected through a cross-sectional household-based survey of centenarian families. The ages of centenarians were verified according to their birth dates from identification cards. The study protocol was approved by the Ethical Committee of the Affiliated Hospital of Nantong University. The survey began in 2020 with the recruiting of centenarian families. We enrolled centenarians, centenarian offspring, and offspring spouses without centenarian parents. By May 6th, 2021, there were 253 centenarians, 217 centenarian offspring, and 116 offspring spouses who signed informed consents and cooperated with a questionnaire interview, physical health examination, and fasting blood sample collection. Among offspring and offspring spouses, there were 101 paired offspring and offspring spouses who lived together, 116 unpaired offspring, and 16 unpaired spouses.

\section{Data Collection}

Age was calculated from the date of birth recorded on identification cards. Health-related behaviors including smoking and alcohol use were collected using a structured questionnaire. Drug use was self-reported. Body mass was measured using an electronic scale and recorded in kilograms. Height and waist circumference were obtained using a flexible tape measure. Waist circumference was measured at the level of the navel. BMI was calculated as mass divided by height squared (kilograms/meters ${ }^{2}$ ). Right-arm blood pressure was checked using a standard electronic arm sphygmomanometer $(1 \mathrm{mmhg}=0.133 \mathrm{kPa})$ under calm conditions. Fasting blood samples were collected by an experienced nurse and placed in a cold box at $4^{\circ} \mathrm{C}$, before transport within 24 hours to the Affiliated Hospital of Nantong University's biobank. Plasma TC, LDL-C, HDL-C, and TG concentrations were assayed using an automated biochemical analyzer. 


\section{Definitions}

Smoking status was classified as never smoker, former smoker, or current smoker. Alcohol status was classified as never drinker, former drinker, and current drinker. Using BMI, we classified participants as underweight (BMI $<18.5 \mathrm{~kg} / \mathrm{m}^{2}$ ), normal (BMI: $18.5-22.9 \mathrm{~kg} / \mathrm{m}^{2}$ ), overweight (BMI: $23.0-27.4 \mathrm{~kg} / \mathrm{m}^{2}$ ), or obese $\left(\right.$ BMI $\geq 27.5 \mathrm{~kg} / \mathrm{m}^{2}$ ) [44]. Central obesity was defined by male waist circumference $>90 \mathrm{~cm}$ or female waist circumference $>85 \mathrm{~cm}$ [45]. Hypertension was defined by systolic blood pressure (SBP) $\geq$ $140 \mathrm{mmHg}$ or diastolic blood pressure (DBP) $\geq 90 \mathrm{mmHg}$ or under hypotensive therapy [46]. According to 2016 Chinese guidelines for managing dyslipidemia in adults, dyslipidemia was identified by meeting one or more of the following criteria: (1) high TC: TC $\geq 6.22 \mathrm{mmol} / \mathrm{L}$ (240 mg/dL), (2) high TG: TG $\geq 2.26 \mathrm{mmol} / \mathrm{L}(200 \mathrm{mg} / \mathrm{dL})$, (3) High LDL-C: LDL-C $\geq 4.14 \mathrm{mmol} / \mathrm{L}(160 \mathrm{mg} / \mathrm{dL})$, (4) Low HDL-C: HDL$\mathrm{C}<1.04 \mathrm{mmol} / \mathrm{L}(40 \mathrm{mg} / \mathrm{dL})$, and (5) under lipid-lowering therapy $[47,48]$.

\section{Statistical Analysis}

Continuous variables were presented as medians with 25 th and 75th percentiles due to skewed distribution. Categorical variables were presented as numbers and proportions. For continuous variables, we performed Kruskal-Wallis tests for comparison of three groups and nonparametric Mann-Whitney $U$ tests for comparison of two groups. Categorical variables were examined using the chi-square test or Fisher's exact test. Bonferroni correction was used for multiple comparisons. We performed multivariate logistic regression on the prevalence of cardiovascular-related factors between groups. The regression model was adjusted for the effects of gender, smoking status, alcohol drinking status, and BMI. The odds ratio (OR) with $95 \%$ confidence intervals was used to measure association. $p<0.05$ were considered statistically significant. All analyses were undertaken using SPSS 23.0 for windows.

\section{Results}

6.1. Demographics, Health-Related Behaviors, Physical Measures, Plasma Lipids, and Drug Use in Centenarian Families. Centenarians had a median age of 100 years, ranging from 99 to 109 years. Centenarian offspring, with a median age of 70 years, and offspring spouses, with a median age of 69 years, were mostly aged beyond 60 years and similar in age. Merely $26.1 \%$ of centenarians were males. Due to Chinese traditional culture, elderly parents prefer to live with their sons rather than their daughters. Thus, there were significantly more males $(70.5 \%)$ in the group of centenarian offspring and more females $(75.0 \%)$ in the group of offspring spouses (Table 1).

For health-related behaviors, both centenarians (15.6\%) and centenarian offspring (19.1\%) had more former smokers than offspring spouses (5.2\%). Meanwhile, centenarian offspring $(25.1 \%)$ had more current smokers than the other two groups. There were more current drinkers among centenarian offspring (48.6\%) than centenarians $(25.7 \%)$ and offspring spouses (28.1\%). Centenarians (14.5\%) had more former drinkers than offspring spouses (5.3\%) (Table 1).

Centenarians displayed lower waist circumference, BMI, and diastolic blood pressure but higher levels of systolic blood pressure than centenarian offspring and offspring spouses $(p<0.05)$. No significant difference in these features was evidenced between centenarian offspring and offspring spouses (Table 1).

Regarding lipid plasma levels, centenarians showed significantly lower levels of TC, LDL-C, and TG than centenarian offspring and offspring spouses $(p<0.05)$. When compared to centenarian offspring, centenarians had low levels of HDL-C $(p<0.05)$. No significant difference in plasma lipids was observed between centenarian offspring and offspring spouses (Table 1).

Regarding the current pharmacologic therapy, a lower percentage $(19.0 \%)$ of centenarians underwent hypotensive therapy than centenarian offspring (30.4\%) and offspring spouses $(37.9 \%)$. Centenarians $(0.0 \%)$ were treated less with lipid-lowering drugs than centenarian offspring (3.7\%). Centenarian offspring and offspring spouses shared similar results on hypotensive and lipid-lowering drug use (Table 1).

\subsection{Prevalence of Cardiovascular-Related Factors between} Centenarian, Centenarian Offspring, and Offspring Spouses. Since the numbers of males and that of females among the three groups are not equal, we compared the prevalence of cardiovascular-related factors among male and female participants. Female centenarians were more likely to have high TC ( $p=0.005)$ than males. No significant difference in prevalence for hypertension, overweight, obesity, central obesity, high LDL-C, low HDL-C, high TG, and dyslipidemia among male and female centenarians was noted (Table 2). Among centenarian offspring, females had a higher prevalence of high TC, high LDL, high TG, and dyslipidemia than males $(p<0.05)$ (Supplementary Table 1$)$. Among offspring spouses, males had a higher prevalence of low HDL than females $(p<0.05)$ (Supplementary Table 2). We did not find a significant difference in other cardiovascular-related factors among male and female participants.

In total, $72.2 \%$ of centenarians suffered hypertension, 9.6\% suffered obesity, $37.7 \%$ suffered central obesity, and $29.2 \%$ suffered dyslipidemia. Low levels of HDL-C were the most common type of dyslipidemia among centenarians $(19.4 \%)$. Then, we considered offspring spouses as the control group. After adjusting for possible confounders such as gender, smoking status, and alcohol drinking status, centenarians had reduced prevalence of overweight (OR 0.336, 95\% CI 0.197-0.572, $p<0.001$ ), obesity (OR $0.245,95 \%$ CI $0.125-0.480, p<0.001$ ), central obesity (OR $0.466,95 \%$ CI $0.285-0.762, p=0.002)$, high TC (OR 0.421, 95\% CI 0.193-0.919, $p=0.030$ ), high LDL-C (OR 0.450, 95\% CI $0.209-0.969, p=0.041$ ), high TG (OR 0.198, 95\% CI $0.077-0.511, p<0.001)$ and greater prevalence of hypertension (OR 2.589, 95\% CI 1.426-4.699, $p=0.002$ ). The difference in prevalence for dyslipidemia was not significant (Table 3). 
Table 1: Demographics, health-related behaviors, physical measures, plasma lipids, and drug use in centenarian families.

\begin{tabular}{|c|c|c|c|c|}
\hline & Centenarian $n=253$ & Centenarian offspring $n=217$ & Offspring spouses $n=116$ & $p$ value \\
\hline \multicolumn{5}{|l|}{ Demographics } \\
\hline Age & $100.0(99.0-101.0)^{\#}$ & $70.0(65.0-75.0)^{*}$ & $69.0(64.5-74.0)$ & $<0.001$ \\
\hline Male, $n(\%)$ & $66(26.1 \%)$ & $153(70.5 \%)^{\# *}$ & $29(25.0 \%)$ & $<0.001$ \\
\hline \multicolumn{5}{|l|}{ Smoking status } \\
\hline Current smoker, $n(\%)$ & $12(4.8 \%)$ & $54(25.1 \%)^{\# *}$ & $12(10.4 \%)$ & $<0.001$ \\
\hline Former smoker, $n(\%)$ & $39(15.6 \%)^{\#}$ & $41(19.1 \%)^{\#}$ & $6(5.2 \%)$ & 0.003 \\
\hline \multicolumn{5}{|l|}{ Alcohol drinking status } \\
\hline Current drinker, $n(\%)$ & $64(25.7 \%)$ & $104(48.6 \%)^{\# *}$ & $32(28.1 \%)$ & $<0.001$ \\
\hline Former drinker, $n(\%)$ & $36(14.5 \%)^{\#}$ & $16(7.5 \%)$ & $6(5.3 \%)$ & 0.007 \\
\hline \multicolumn{5}{|l|}{ Physical measures } \\
\hline Waist circumference $(\mathrm{cm})$ & $84.0(76.0-90.0)^{\#}$ & $87.0(80.0-93.0)^{*}$ & $88.0(81.0-97.0)$ & 0.001 \\
\hline BMI $\left(\mathrm{kg} / \mathrm{m}^{2}\right)$ & $21.12(18.79-23.73)^{\#}$ & $24.25(22.29-26.58)^{*}$ & $24.90(22.26-27.75)$ & $<0.001$ \\
\hline $\mathrm{SBP}(\mathrm{mmHg})$ & $150(132-169)^{\#}$ & $141(126-157)^{*}$ & $140(124-159)$ & $<0.001$ \\
\hline DBP (mmHg) & $72(64-82)^{\#}$ & $80(73-87)^{*}$ & $77(71-85)$ & $<0.001$ \\
\hline \multicolumn{5}{|l|}{ Lipids } \\
\hline $\mathrm{TC}(\mathrm{mmol} / \mathrm{L})$ & $4.6(4.1-5.3)^{\#}$ & $5.1(4.5-5.8)^{*}$ & $5.2(4.5-5.8)$ & $<0.001$ \\
\hline LDL-C (mmol/L) & $2.89(2.47-3.35)^{\#}$ & $3.26(2.77-3.78)^{*}$ & $3.34(2.85-3.84)$ & $<0.001$ \\
\hline HDL-C (mmol/L) & $1.32(1.09-1.53)$ & $1.36(1.20-1.60)^{*}$ & $1.36(1.13-1.55)$ & 0.049 \\
\hline $\mathrm{TG}(\mathrm{mmol} / \mathrm{L})$ & $1.02(0.85-1.37)^{\#}$ & $1.15(0.86-1.63)^{*}$ & $1.24(0.87-1.87)$ & 0.002 \\
\hline \multicolumn{5}{|l|}{ Drug use } \\
\hline Hypotensive therapy, $n(\%)$ & $48(19.0 \%)^{\#}$ & $66(30.4 \%)^{*}$ & $44(37.9 \%)$ & $<0.001$ \\
\hline Lipid-lowering therapy, $n$ (\%) & $0(0.0 \%)$ & $8(3.7 \%)^{*}$ & $1(0.9 \%)$ & 0.002 \\
\hline
\end{tabular}

$n$, number; \%, percentage; BMI, body mass index; SBP, systolic blood pressure; DBP, diastolic blood pressure; TC, total cholesterol; LDL-C, low-density lipoprotein cholesterol; HDL-C, high-density lipoprotein cholesterol; TG, triglycerides. "Bonferroni-corrected $p<0.05$ versus offspring spouses. *Bonferroni-corrected $p<0.05$ versus centenarians.

TABle 2: Prevalence of cardiovascular-related factors in centenarians stratified by gender.

\begin{tabular}{lccc}
\hline & Male & Female & $p$ value \\
\hline Hypertension, $n(\%)$ & $43(68.3 \%)$ & $131(73.6 \%)$ & 0.416 \\
Overweight, $n(\%)$ & $17(28.3 \%)$ & $26(17.7 \%)$ & 0.087 \\
Obesity, $n(\%)$ & $9(15.0 \%)$ & $11(7.4 \%)$ & 0.093 \\
Central obesity, $n(\%)$ & $19(32.8 \%)$ & $56(39.7 \%)$ & 0.357 \\
High TC, $n(\%)^{*}$ & $0(0.0 \%)$ & $19(10.2 \%)$ & 0.005 \\
High LDL-C, $n(\%)^{*}$ & $2(3.0 \%)$ & $18(9.6 \%)$ & 0.088 \\
Low HDL-C, $n(\%)^{*}$ & $17(25.8 \%)$ & $32(17.1 \%)$ & 0.127 \\
High TG, $n(\%)^{*}$ & $3(4.5 \%)$ & $8(4.3 \%)$ & 1.000 \\
Dyslipidemia, $n(\%)$ & $19(28.8 \%)$ & $55(29.4 \%)$ & 0.924 \\
\hline
\end{tabular}

$n$, number; \%, percentage; TC, total cholesterol; LDL-C, low-density lipoprotein cholesterol; HDL-C, high-density lipoprotein cholesterol; TG, triglycerides. * Subjects under lipid-lowering agents were excluded from the analysis.

However, centenarian offspring and offspring spouses shared similar risks of hypertension, overweight, obesity, central obesity, high TC, high LDL-C, low HDL-C, high TG, and dyslipidemia (Table 4). Furthermore, comparison between subgroups, including paired offspring and offspring spouses who lived together, unpaired offspring, and unpaired spouses, found no significant difference in cardiovascular-related factors (Supplementary Table 3).

\section{Discussion}

Centenarians are recognized as the product of their personal genetics $\times$ environment equation and human longevity clusters in families [5]. However, few studies focus on centenarian families where centenarians, centenarian offspring, and offspring spouses live in the same environment. In our study, we recruited centenarian families in Rugao and controlled for the geographic and cultural environmental difference. Furthermore, we enrolled paired centenarian offspring and offspring spouses within the same familial environment.

We found that centenarians had lower prevalence of overweight, obesity, central obesity, high TC, high LDL-C, and high TG than elderly controls, as previously reported in Hainan and Portuguese centenarians [13, 36]. Also, decreased TC, LDL-C, and TG concentrations were considered as possibly beneficial factors preventing centenarians from cardiovascular diseases and promoting longevity [13]. However, centenarian offspring did not present a similar profile as their centenarian parents. In our study, they shared similar risks of hypertension, obesity, central obesity, and dyslipidemia with offspring spouses. In the absence of data on venous blood glucose, the prevalence of diabetes was not analyzed in our study. A previous study on Italian centenarian offspring reported a similar prevalence of diabetes between centenarian offspring and controls of non-longlived parents, while American and Ashkenazi Jewish centenarian offspring were reported with a lower prevalence of diabetes [26, 37, 49]. The discrepancy was thought to be associated with the environmental context, cultural habits, and lifestyle factors [37].

In our study, female centenarians had a greater prevalence of high TC than males, and sex differentials on lipid profiles were also discovered among centenarian offspring and offspring spouses. In general population, male is a risk factor for dyslipidemia [47]. But with the increase of age, the sex difference on lipid concentrations narrows, and 
TABLE 3: Prevalence of cardiovascular-related factors between centenarians and offspring spouses.

\begin{tabular}{|c|c|c|c|c|}
\hline & Centenarian & Offspring spouses & OR $(95 \% \mathrm{CI})$ & $p$ value \\
\hline Hypertension, $n(\%)^{\mathrm{a}}$ & $174(72.2 \%)$ & $67(59.8 \%)$ & $2.589(1.426-4.699)$ & 0.002 \\
\hline Overweight, $n(\%)^{\mathrm{b}}$ & $43(20.8 \%)$ & $47(42.3 \%)$ & $0.336(0.197-0.572)$ & $<0.001$ \\
\hline Obesity, $n(\%)^{\mathrm{b}}$ & $20(9.6 \%)$ & $31(27.7 \%)$ & $0.245(0.125-0.480)$ & $<0.001$ \\
\hline Central obesity, $n(\%)^{\mathrm{b}}$ & $75(37.7 \%)$ & $62(55.9 \%)$ & $0.466(0.285-0.762)$ & 0.002 \\
\hline High TC, $n(\%)^{* \mathrm{a}}$ & $19(7.5 \%)$ & $21(18.3 \%)$ & $0.421(0.193-0.919)$ & 0.030 \\
\hline High LDL-C, $n(\%)^{* \mathrm{a}}$ & $20(7.9 \%)$ & $21(18.3 \%)$ & $0.450(0.209-0.969)$ & 0.041 \\
\hline Low HDL-C, $n(\%)^{* \mathrm{a}}$ & $49(19.4 \%)$ & $10(8.7 \%)$ & $2.629(1.140-6.063)$ & 0.023 \\
\hline High TG, $n(\%)^{* \mathrm{a}}$ & $11(4.3 \%)$ & $22(19.1 \%)$ & $0.198(0.077-0.511)$ & $<0.001$ \\
\hline Dyslipidemia, $n(\%)^{\mathrm{a}}$ & $74(29.2 \%)$ & $39(33.6 \%)$ & $0.885(0.508-1.539)$ & 0.664 \\
\hline
\end{tabular}

$n$, number; \%, percentage; OR, odds ratio; CI, confidence interval; TC, total cholesterol; LDL-C, low-density lipoprotein cholesterol; HDL-C, high-density

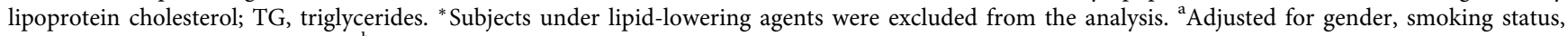
alcohol drinking status, and BMI. ${ }^{\mathrm{b}}$ Adjusted for gender, smoking status, and alcohol drinking status.

TABLe 4: Prevalence of cardiovascular-related factors between centenarian offspring and offspring spouses.

\begin{tabular}{|c|c|c|c|c|}
\hline & Centenarian offspring & Offspring spouses & OR $(95 \% \mathrm{CI})$ & $p$ value \\
\hline Hypertension, $n(\%)^{\mathrm{a}}$ & $133(62.1 \%)$ & $67(59.8 \%)$ & $1.343(0.763-2.364)$ & 0.307 \\
\hline Overweight, $n(\%)^{\mathrm{b}}$ & $103(49.8 \%)$ & $47(42.3 \%)$ & $1.380(0.811-2.348)$ & 0.235 \\
\hline Obesity, $n(\%)^{\mathrm{b}}$ & $37(17.8 \%)$ & $31(27.7 \%)$ & $0.575(0.305-1.086)$ & 0.088 \\
\hline Central obesity, $n(\%)^{\mathrm{b}}$ & $89(42.4 \%)$ & $62(55.9 \%)$ & $0.600(0.354-1.016)$ & 0.057 \\
\hline High TC, $n(\%)^{* \mathrm{a}}$ & $28(13.4 \%)$ & $21(18.3 \%)$ & $1.291(0.634-2.628)$ & 0.481 \\
\hline High LDL-C, $n(\%)^{* \mathrm{a}}$ & $30(14.4 \%)$ & $21(18.3 \%)$ & $1.208(0.597-2.443)$ & 0.600 \\
\hline Low HDL-C, $n(\%)^{* \mathrm{a}}$ & $21(10.0 \%)$ & $10(8.7 \%)$ & $1.188(0.450-3.138)$ & 0.728 \\
\hline High TG, $n(\%)^{* \mathrm{a}}$ & $24(11.5 \%)$ & $22(19.1 \%)$ & $0.991(0.477-2.059)$ & 0.982 \\
\hline Dyslipidemia, $n(\%)^{\mathrm{a}}$ & $65(30.0 \%)$ & $39(33.6 \%)$ & $1.243(0.699-2.212)$ & 0.459 \\
\hline
\end{tabular}

$n$, number; \%, percentage; OR, odds ratio; CI, confidence interval; TC, total cholesterol; LDL-C, low-density lipoprotein cholesterol; HDL-C, high-density

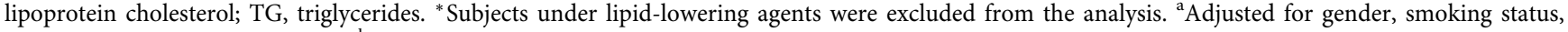
alcohol drinking status, and BMI. ${ }^{\mathrm{b}}$ Adjusted for gender, smoking status, and alcohol drinking status.

menopause contributes to increased levels of TC, LDL-C, and TG, and decreased levels of HDL-C in women is associated with reduced oestrogen, which may lead to higher TC levels in female centenarians $[47,50,51]$. Besides, centenarians and centenarian offspring appeared to have larger percentages of smokers and alcohol drinkers than offspring spouses. Also, the effects of cigarette smoking and alcohol drinking on CVDs have been discovered in previous studies $[52,53]$. Thus, we performed multivariate analysis on the prevalence of cardiovascular-related factors adjusting for the effects of gender, smoking status, and alcohol drinking status.

Cardiovascular-related factors such as BMI, blood pressure, and lipids are influenced by multiple factors. Although we recruited participants residing in the same geographic, cultural, and familial area, they may adopt different diets and behaviors, which substantially affect risks of obesity, hypertension, and dyslipidemia [16-18]. Obesity is an independent risk factor for cardiovascular diseases [10]. In our study, centenarian offspring and offspring spouses shared similar BMI and prevalence of overweight and obesity. Our finding may reflect a weaker genetic effect and a stronger environmental effect on BMI. Twin and adoption studies revealed a $50 \%-80 \%$ of heritability on obesity $[54,55]$. However, genetic studies demonstrate a weak explanation by genetic variance [56]. A longitudinal study in Norway implies environment is the main contributor to BMI [55]. Centenarians had lower waist circumference, BMI, and prevalence of overweight and obesity, corresponding to other research on centenarians $[35,36,57]$. However, these features may result from lower energy intake and frailty $[34,35]$.

Higher blood pressure and LDL-C contribute to adverse cardiometabolic events among elderly people [58]. In our study, we did not find a significant difference between centenarian offspring and offspring spouses regarding blood pressure and plasma lipids, in contrast with studies reporting a lower prevalence of hypertension and hypercholesterolemia among centenarian offspring than age-matched controls $[37,39,40]$. Similar research on centenarian offspring which recruited spouses as controls found no significant difference in the prevalence of hypertension and new onset of hypertension during follow-up [25]. These findings may indicate a greater impact of environment on hypertension than genetic factors. The effect of environmental factors may also contribute to lipid result interpretation [38, 47, 59]. However, future studies are needed to clarify the differential results across studies. Centenarians had a higher prevalence of hypertension, which corresponds with previous studies revealing the high prevalence of age-related diseases in centenarians such as hypertension, stroke, and heart disease $[35,60]$. As for lipids, centenarians presented lower levels of TC, LDL-C, and TG. Whether this lipid profile among centenarians is a predictor of exceptional longevity or it is a consequence of aging is still unclear [31,33, 57]. No significant difference was found concerning HDL-C levels between centenarians and offspring spouses. HDL-C was strongly associated with genetic factors and generally 
decreases with age $[61,62]$. Additionally, HDL-C has been considered as a more important and protective factor in elderly people [61]. Thus, the reserve of HDL-C levels in centenarians may be associated with the longevity phenotype.

However, there are several limitations in this study. First, our cross-sectional study is based on centenarian families in Rugao, which fails to represent all centenarian families, and longitudinal studies are needed to provide stronger evidence. Second, possible confounders such as diet, physical activity, sleep condition, and other cardiovascular-related factors such as fasting blood glucose and history of CVDs were not analyzed. Finally, further studies are needed to determine genetic and environmental effects on longevity and CVDs.

\section{Conclusions}

In conclusion, centenarians and centenarian offspring did not present significantly improved blood pressure, plasma lipids, or BMI in comparison with offspring spouses. Further research on longevity and cardiovascular diseases are recommended to elucidate contributing factors to CVD development and cultivate successful intervention strategies.

\section{Data Availability}

The raw data required to reproduce these findings cannot be shared at this time as the data also forms part of an ongoing study.

\section{Ethical Approval}

Ethical approval was obtained from the ethical committee of the Affiliated Hospital of Nantong University.

\section{Consent}

Informed consent was obtained from all individual participants included in the study.Participants signed informed consent regarding publishing their data.

\section{Conflicts of Interest}

The authors declare no conflicts of interest.

\section{Authors' Contributions}

All authors contributed to the study conception and design. The data assembly was performed by Rui Zhao and Chen Dong. Data analysis was performed by Peng-Cheng Hai. The first draft of the manuscript was written by Peng-Cheng Hai, and all authors commented on previous versions of the manuscript. All authors read and approved the final manuscript.

\section{Acknowledgments}

This study was funded by the Beijing Outstanding Young Scientist Program (No. BJJWZYJH01201910025030). The authors would like to thank all the reviewers who participated in the review.

\section{Supplementary Materials}

Supplementary Table 1: prevalence of cardiovascular-related factors in centenarian offspring stratified by gender. Supplementary Table 2: prevalence of cardiovascular-related factors in offspring spouses stratified by gender. Supplementary Table 3: prevalence of cardiovascular-related factors between subgroups. (Supplementary Materials)

\section{References}

[1] G. A. Roth, G. A. Mensah, C. O. Johnson et al., "Global burden of cardiovascular diseases and risk factors, 1990-2019: update from the GBD 2019 study," Journal of the American College of Cardiology, vol. 76, no. 25, pp. 2982-3021, 2020.

[2] V. Fuster, "Global burden of cardiovascular disease: time to implement feasible strategies and to monitor results," Journal of the American College of Cardiology, vol. 64, no. 5, pp. 520-522, 2014.

[3] S. Yusuf, S. Hawken, S. Ônpuu et al., "Effect of potentially modifiable risk factors associated with myocardial infarction in 52 countries (the interheart study): case-control study," The Lancet, vol. 364, no. 9438, pp. 937-952, 2004.

[4] D. M. Lloyd-Jones, Y. Hong, D. Labarthe et al., "Defining and setting national goals for cardiovascular health promotion and disease reduction: the American Heart Association's strategic Impact Goal through 2020 and beyond," Circulation, vol. 121, no. 4, pp. 586-613, 2010.

[5] C. Franceschi, P. Garagnani, F. Olivieri, S. Salvioli, and C. Giuliani, "The contextualized genetics of human longevity: JACC focus seminar," Journal of the American College of Cardiology, vol. 75, no. 8, pp. 968-979, 2020.

[6] S. Q. Ye and P. O. Kwiterovich, "Influence of genetic polymorphisms on responsiveness to dietary fat and cholesterol," The American Journal of Clinical Nutrition, vol. 72, pp. 1275S-1284S, 2000.

[7] A. T. Kraja, D. I. Chasman, K. E. North et al., "Pleiotropic genes for metabolic syndrome and inflammation," Molecular Genetics and Metabolism, vol. 112, no. 4, pp. 317-338, 2014.

[8] D. Corella, P. Carrasco, J. V. Sorlí et al., "Mediterranean diet reduces the adverse effect of the TCF7L2-rs7903146 polymorphism on cardiovascular risk factors and stroke incidence: a randomized controlled trial in a high-cardiovascular-risk population," Diabetes Care, vol. 36, no. 11, pp. 3803-3811, 2013.

[9] D. Welter, J. MacArthur, J. Morales et al., "The NHGRI GWAS Catalog, a curated resource of SNP-trait associations," Nucleic Acids Research, vol. 42, no. D1, pp. D1001-D1006, 2014.

[10] M. M. Y. Sung and J. R. B. Dyck, "Age-related cardiovascular disease and the beneficial effects of calorie restriction," Heart Failure Reviews, vol. 17, no. 4-5, pp. 707-719, 2012.

[11] A. J. Walley, A. I. F. Blakemore, and P. Froguel, "Genetics of obesity and the prediction of risk for health," Human Molecular Genetics, vol. 15, no. suppl_2, pp. R124-R130, 2006.

[12] N. Savji, C. B. Rockman, A. H. Skolnick et al., "Association between advanced age and vascular disease in different arterial territories: a population database of over 3.6 million subjects," Journal of the American College of Cardiology, vol. 61, no. 16, pp. 1736-1743, 2013. 
[13] Y.-H. He, S.-Y. Pu, F.-H. Xiao et al., "Improved lipids, diastolic pressure and kidney function are potential contributors to familial longevity: a study on 60 Chinese centenarian families," Scientific Reports, vol. 6, no. 1, Article ID 21962, 2016.

[14] F. Crea, "Prevention of cardiovascular diseases and dementia: the emerging role of air pollution, socioeconomic factors, e-cigarettes, new biomarkers, proteomics, and genetics," European Heart Journal, vol. 41, no. 41, pp. 3969-3972, 2020.

[15] T. Münzel, M. Sørensen, J. Lelieveld et al., "Heart healthy cities: genetics loads the gun but the environment pulls the trigger," European Heart Journal, vol. 42, no. 25, pp. 2422-2438, 2021.

[16] E. A. O’Connor, C. V. Evans, M. C. Rushkin, N. Redmond, and J. S. Lin, "Behavioral counseling to promote a healthy diet and physical activity for cardiovascular disease prevention in adults with cardiovascular risk factors: updated evidence report and systematic review for the US preventive services task force," JAMA, vol. 324, pp. 2076-2094, 2020.

[17] P. K. Whelton, R. M. Carey, W. S. Aronow et al., "ACC/AHA/ AAPA/ABC/ACPM/AGS/APhA/ASH/ASPC/NMA/PCNA guideline for the prevention, detection, evaluation, and management of high blood pressure in adults: a report of the American college of cardiology/American heart association task force on clinical practice guidelines," Journal of the American College of Cardiology, vol. 71, pp. e127-e248, 2017.

[18] C. J. Lavie, D. Laddu, R. Arena, F. B. Ortega, M. A. Alpert, and R. F. Kushner, "Healthy weight and obesity prevention: JACC health promotion series," Journal of the American College of Cardiology, vol. 72, no. 13, pp. 1506-1531, 2018.

[19] P. Sebastiani, N. Solovieff, A. T. Dewan et al., "Genetic signatures of exceptional longevity in humans," PLoS One, vol. 7, no. 1, Article ID e29848, 2012.

[20] G. Muntané, X. Farré, J. A. Rodríguez et al., "Biological processes modulating longevity across primates: a phylogenetic genome-phenome analysis," Molecular Biology and Evolution, vol. 35, no. 8, pp. 1990-2004, 2018.

[21] L. C. Pilling, C.-L. Kuo, K. Sicinski et al., "Human longevity: 25 genetic loci associated in 389,166 UK biobank participants," Aging, vol. 9, no. 12, pp. 2504-2520, 2017.

[22] K. Fortney, E. Dobriban, P. Garagnani et al., "Genome-Wide scan informed by age-related disease identifies loci for exceptional human longevity," PLoS Genetics, vol. 11, no. 12, Article ID e1005728, 2015.

[23] C. Giuliani, M. Sazzini, C. Pirazzini et al., "Impact of demography and population dynamics on the genetic architecture of human longevity," Aging, vol. 10, no. 8, pp. 1947-1963, 2018.

[24] P. Gueresi, R. Miglio, D. Monti et al., "Does the longevity of one or both parents influence the health status of their offspring?" Experimental Gerontology, vol. 48, no. 4, pp. 395-400, 2013.

[25] E. R. Adams, V. G. Nolan, S. L. Andersen, T. T. Perls, and D. F. Terry, "Centenarian offspring: start healthier and stay healthier," Journal of the American Geriatrics Society, vol. 56, no. 11, pp. 2089-2092, 2008.

[26] D. F. Terry, M. A. Wilcox, M. A. McCormick, and T. T. Perls, "Cardiovascular disease delay in centenarian offspring," The Journals of Gerontology. Series A, Biological Sciences and Medical Sciences, vol. 59, pp. 385-389, 2004.

[27] D. Albani, S. Mazzuco, L. Polito et al., "Insulin-like growth factor 1 receptor polymorphism rs2229765 and circulating interleukin-6 level affect male longevity in a population-based prospective study (treviso longeva- trelong)," The Aging Male, vol. 14, no. 4, pp. 257-264, 2011.

[28] F. Flachsbart, J. Dose, L. Gentschew et al., "Identification and characterization of two functional variants in the human longevity gene FOXO3," Nature Communications, vol. 8, no. 1, p. 2063, 2017

[29] Y. Zeng, C. Nie, J. Min et al., "Novel loci and pathways significantly associated with longevity," Scientific Reports, vol. 6, no. 1, Article ID 21243, 2016.

[30] R. Richmond, J. Law, and F. Kay-Lambkin, "Higher blood pressure associated with higher cognition and functionality among centenarians in Australia," American Journal of $\mathrm{Hy}$ pertension, vol. 24, no. 3, pp. 299-303, 2011.

[31] S. S. Wang, S. S. Yang, W. P. Jia et al., "Distribution characteristics of blood lipid profile in Hainan centenarians," Zhonghua Liuxingbingxue Zazhi, vol. 42, pp. 80-87, 2021.

[32] I. Pradas, M. Jové, K. Huynh et al., "Exceptional human longevity is associated with a specific plasma phenotype of ether lipids," Redox Biology, vol. 21, Article ID 101127, 2019.

[33] D. B. Hausman, J. G. Fischer, and M. A. Johnson, "Protein, lipid, and hematological biomarkers in centenarians: definitions, interpretation and relationships with health," Maturitas, vol. 71, no. 3, pp. 205-212, 2012.

[34] Y.-J. Zhang, S.-H. Fu, Q. Zhu et al., "Underweight in men had a closer relationship with falls than women in centenarians," The Journal of Nutrition, Health \& Aging, vol. 24, no. 9, pp. 987-992, 2020.

[35] P. Martin, Y. Gondo, Y. Arai et al., "Cardiovascular health and cognitive functioning among centenarians: a comparison between the Tokyo and Georgia centenarian studies," International Psychogeriatrics, vol. 31, no. 4, pp. 455-465, 2019.

[36] S. A. Da, A. Valente, C. Chaves et al., "Characterization of Portuguese centenarian eating habits, nutritional biomarkers, and cardiovascular risk: a case control study," Oxidative Medicine and Cellular Longevity, vol. 2018, Article ID 5296168, 10 pages, 2018.

[37] L. Bucci, R. Ostan, E. Cevenini et al., “Centenarians' offspring as a model of healthy aging: a reappraisal of the data on Italian subjects and a comprehensive overview," Aging, vol. 8, no. 3, pp. 510-519, 2016.

[38] R. Ostan, L. Bucci, E. Cevenini et al., "Metabolic syndrome in the offspring of centenarians: focus on prevalence, components, and adipokines," Age, vol. 35, no. 5, pp. 1995-2007, 2013.

[39] A. A. M. Vaarhorst, M. Beekman, M. Beekman et al., "Lipid metabolism in long-lived families: the leiden longevity study," Age, vol. 33, no. 2, pp. 219-227, 2011.

[40] M. P. Rozing, R. G. J. Westendorp, A. J. M. de Craen et al., "Favorable glucose tolerance and lower prevalence of metabolic syndrome in offspring without diabetes mellitus of nonagenarian siblings: the Leiden longevity study," Journal of the American Geriatrics Society, vol. 58, no. 3, pp. 564-569, 2010.

[41] N. Barzilai, I. Gabriely, M. Gabriely, N. Iankowitz, and J. D. Sorkin, "Offspring of centenarians have a favorable lipid profile," Journal of the American Geriatrics Society, vol. 49, no. 1, pp. 76-79, 2001.

[42] M.-J. Favé, F. C. Lamaze, D. Soave et al., "Gene-by-environment interactions in urban populations modulate risk phenotypes," Nature Communications, vol. 9, no. 1, p. 827, 2018.

[43] Z. Liu, Y. Wang, Y. Zhang et al., "Cohort profile: the Rugao longevity and ageing study (RuLAS)," International Journal of Epidemiology, vol. 45, pp. 1064-1073, 2016. 
[44] WHO Expert Consultation, “Appropriate body-mass index for Asian populations and its implications for policy and intervention strategies," Lancet, vol. 363, pp. 157-163, 2004.

[45] Y. Bao, J. Lu, C. Wang et al., "Optimal waist circumference cutoffs for abdominal obesity in Chinese," Atherosclerosis, vol. 201, no. 2, pp. 378-384, 2008.

[46] Q. Hua, L. Fan, and J. Li, "Chinese guideline for the management of hypertension in the elderly," Journal of Geriatric Cardiology, vol. 16, pp. 67-99, 2019.

[47] P. Song, M. Zha, X. Yang et al., "Socioeconomic and geographic variations in the prevalence, awareness, treatment and control of dyslipidemia in middle-aged and older Chinese," Atherosclerosis, vol. 282, pp. 57-66, 2019.

[48] C. F. G. R. Joint, "2016 Chinese guidelines for the management of dyslipidemia in adults," Journal of Geriatric Cardiology: JGC, vol. 15, pp. 1-29, 2018.

[49] G. Atzmon, C. Schechter, W. Greiner, D. Davidson, G. Rennert, and N. Barzilai, "Clinical phenotype of families with longevity," Journal of the American Geriatrics Society, vol. 52, no. 2, pp. 274-277, 2004.

[50] K. S. Reddy and S. R. Chandala, "A comparative study of lipid profile and oestradiol in pre- and post-menopausal women," Journal of Clinical and Diagnostic Research, vol. 7, pp. 1596-1598, 2013.

[51] D. S. Freedman, J. D. Otvos, E. J. Jeyarajah et al., "Sex and age differences in lipoprotein subclasses measured by nuclear magnetic resonance spectroscopy: the Framingham Study," Clinical Chemistry, vol. 50, no. 7, pp. 1189-1200, 2004.

[52] B. Messner and D. Bernhard, "Smoking and cardiovascular disease: mechanisms of endothelial dysfunction and early atherogenesis," Arteriosclerosis, Thrombosis, and Vascular Biology, vol. 34, no. 3, pp. 509-515, 2014.

[53] E. Degerud, I. Ariansen, E. Ystrom et al., "Life course socioeconomic position, alcohol drinking patterns in midlife, and cardiovascular mortality: analysis of Norwegian population-based health surveys," PLoS Medicine, vol. 15, no. 1, Article ID e1002476, 2018.

[54] B. Rokholm, K. Silventoinen, P. Tynelius, M. Gamborg, T. I. A. Sørensen, and F. Rasmussen, "Increasing genetic variance of body mass index during the Swedish obesity epidemic," PLoS One, vol. 6, no. 11, Article ID e27135, 2011.

[55] M. Brandkvist, J. H. Bjørngaard, R. A. Ødegård, B. O. Åsvold, E. R. Sund, and G. A. Vie, "Quantifying the impact of genes on body mass index during the obesity epidemic: longitudinal findings from the HUNT Study," BMJ, vol. 366, Article ID 14067, 2019.

[56] L. Yengo, J. Sidorenko, K. E. Kemper et al., "Meta-analysis of genome-wide association studies for height and body mass index in 700000 individuals of European ancestry," Human Molecular Genetics, vol. 27, no. 20, pp. 3641-3649, 2018.

[57] M. Liu, F. Kou, S. Yang, S. Wang, Y. He, and W. Zhang, "Ideal cardiovascular health in the oldest-old and centenarians and its association with disability and health-related quality of life," Frontiers in Cardiovascular Medicine, vol. 8, Article ID 603877, 2021.

[58] W. Lu, H. Pikhart, and A. Sacker, "Domains and measurements of healthy aging in epidemiological studies: a review," The Gerontologist, vol. 59, no. 4, pp. e294-e310, 2019.

[59] C.-F. Tsao, C.-M. Chang, S.-W. Weng, P.-W. Wang, C.-Y. Lin, and S.-N. Lu, "Identifying endemic areas and estimating the prevalence of hyperlipidemia in Taiwan's townships," Journal of the Formosan Medical Association, vol. 120, no. 1, pp. 460-465, 2021.
[60] J. Zyczkowska, A. Klich-Raczka, M. Mossakowska, J. Gasowski, K. Wieczorowska-Tobis, and T. Grodzicki, "Blood pressure in centenarians in Poland," Journal of Human Hypertension, vol. 18, pp. 713-716, 2004.

[61] E. Windler, M. Schöffauer, and B.-C. Zyriax, "The significance of low HDL-cholesterol levels in an ageing society at increased risk for cardiovascular disease," Diabetes and Vascular Disease Research, vol. 4, no. 2, pp. 136-142, 2007.

[62] Y. Arai and N. Hirose, "Aging and HDL metabolism in elderly people more than 100 years old," Journal of Atherosclerosis and Thrombosis, vol. 11, no. 5, pp. 246-252, 2004. 